\title{
Cardiovascular risk stratification among type 2 diabetes mellitus
}

\section{Opinion}

With rising prevalence of type 2 Diabetes Mellitus (T2DM), burden of cardiovascular diseases is also rising at rapid pace. T2DM individuals are at two to six times' high risk of coronary artery disease (CAD) and stroke than non-diabetics. Their risk of developing cardiovascular disease (CVD) is equivalent to non-diabetics with previous CAD ${ }^{1} \mathrm{~A}$ coronary artery disease is the leading cause of morbidity and mortality among T2DM patients. Hence even if individuals with T2DM are at higher risk of developing cardiovascular complications, their risk stratification plays important role in therapeutic modifications in their management. T2DM and CVD, both are chronic metabolic disorders sharing common multiple risk factors. Precise risk assessment will help the clinicians to target the high-risk populations with most appropriate preventive measures. Cardiovascular risk stratification of diabetics has also prognostic significance during management of CVD events. T2DM patients are at high risk of developing CAD at an earlier age than non-diabetic counterparts. They remain undiagnosed because of silent ischemia and present with extensive diffuse damage to coronary arteries leading to poor long-term outcome. ${ }^{2}$ Framingham risk score (FRS) calculator is widely used and most popular tool to predict 10year cardiovascular risk. But recently it has been stated that FRS cannot be used to stratify cardiovascular (CV) risk of diabetics because T2DM itself is a coronary disease equivalent. During past two decades various risk tools were developed by the researchers to stratify T2DM patients' cardiovascular risk using various traditional $\mathrm{CV}$ risk factors. FRS was the first tool used to predict next 10year risk of CAD based on findings from Framingham study of Caucasians participants. But this scale, because of inclusion of variable presence of T2DM, which is proposed as CAD equivalent, could not be found useful for diabetics. UK Prospective Diabetes Study (UKPDS) is a diabetes-specific risk engine, which incorporated duration of DM and glycemic control along with traditional cardiac risk factors. ${ }^{3}$ For south Asian people, world health organization/ International society of Hypertension (WHO/ISH) tool was designed which included five parameters- age, sex, systolic blood pressure, smoking, and total cholesterol. But this tool excluded very important major risk factorsdiastolic blood pressure and low-density lipoproteins (LDL). Study from Sri lanka observed significant discrepancy between WHO/ ISH and UKPDS risk engines and poor sensitivity of both among diabetics. $^{4}$

Hernáez R et al. ${ }^{5}$ compared coronary risk in Spanish T2DM subjects using Spanish scale REGICOR and UKPDS risk engines. REGICOR equation included variables sex, age, presence or absence of T2DM, smoking, systolic a diastolic blood pressures, total cholesterol and high density cholesterol levels for risk assessment. UKPDs risk engine included age, sex, ethnic group, systolic blood pressure, smoking, duration of diabetes, total cholesterol, HDL, glycosylated haemoglobin $(\mathrm{HbAlc})$ and presence of atrial fibrillation. They observed 10year coronary risk was more with UKPDS engine than REGICOR equation scale $(15.7+/-8.4 \%$ versus $5.8+/-2.5 \%$ respectively). This signifies the importance of duration of diabetes, glycemic status and presence of atrial fibrillation in risk stratification
Volume 3 Issue 4 - 2016

\author{
Shilpa Balaji Asegaonkar \\ Department of Biochemistry, Government Medical College, \\ India
}

Correspondence: Shilpa Balaji Asegaonkar, Department of Biochemistry, Government Medical College, Maharashtra, India, Tel 9420763 430,Email b_asegaonkar@yahoo.coms

Received: July 30, 2016 | Published: August 0I, 2016

of T2DM patients. ${ }^{5}$ Brazilian study reported high risk of CHD ( $>20 \%$ in 10years) and risk more among males in comparison with females. They estimated CV risk by recording variables age, sex, blood pressure, duration of diabetes, body mass index, lipid profile parameters, fasting and postprandial blood glucose levels, HbAlc, microalbuminuria, fundoscopy and presence or absence of diabetic foot among 1382 T2DM participants. In this cohort, estimated CV risk correlated with lipid profile and microvascular complications, but weakly glycemic control. ${ }^{6}$ This emphasizes the significance of aggressive management of non-glycemic cardiovascular risk factors along with glycemic control. This includes control of hypertension, use of aspirin and lipid lowering drugs depending upon $\mathrm{CV}$ risk score. Along with pharmacological management, therapeutic lifestyle change with behaviour modification, self-management support and long term follow up for control of glycemic status and CV risk is required.

Role of various emerging non-traditional novel $\mathrm{CV}$ risk factors has been widely studied in the development of CVD among diabetics. One of the study included estimation of 23 biomarkers to improve prediction of CV risk in 1002 T2DM subjects from SMART and EPIC-NL study. They found combination of N-terminal prohormane B type natriuretic peptide; osteopontin and MMP3 improved CV risk estimation than traditional risk factors. ${ }^{7}$ But novel biomarkers estimation for $\mathrm{CV}$ risk prediction has limitation of financial constrain especially in developing and undeveloped countries. Findings of the meta-analysis and systematic review by Bulugahapitiya and associates did not support the hypothesis that says diabetes is equivalent to CAD. Patients with T2DM without previous myocardial infarction had $43 \%$ less risk of developing CAD than non-diabetics with myocardial infarction. ${ }^{8}$ Myocardial perfusion scintigraphy and coronary artery calcium imaging are also used and found to be superior to conventional cardiac risk factors to detect subclinical CAD and improve risk stratification among T2DM patients. ${ }^{9}$ But availability of the techniques and cost effectiveness are limiting factors for including them as routine screening tools.

CVD accounts for substantial morbidity and mortality among T2DM patients. More than $65 \%$ of diabetics die because of CVD. ${ }^{10}$ Hence while managing T2DM clinicians should target aggressively $\mathrm{CV}$ risk factors along with glossocentric treatment. T2DM is a complicated clinical entity that significantly affects quality of life 
and survival of the patients particularly with respect to cardiac complications. So early screening and identification of high risk individuals and implementing aggressive preventive interventions will minimize the burden of the disease. Risk estimation will become more relevant if risk scales developed with modifiable, feasible, cost effective, sensitive, validated variables for appropriate population.

\section{Acknowledgements}

None.

\section{Conflict of interest}

Author declares that there is no conflict of interest.

\section{References}

1. Haffner SM, Lehto S, Ronnemaa T, et al. Mortality from coronary heart disease in subjects with type 2 diabetes and in nondiabetic subjects with and without prior myocardial infarction. NEJM. 1998;339:229-234.

2. Cuocolo A, Concilio C, Acampa W, et al. Cardiovascular risk stratification of diabetic patients. Minerva Endocrinol. 2009;34(3):205-221.

3. Chamnan P, Simmons RK, Sharp SJ, et al. Cardiovascular risk assessment scores for people with diabetes: a systematic review $s 2009 ; 52(10): 2001-2014$.
4. Rubén Hernáez, Lucía Choque, Margarita Giménez, et al. Coronary Risk Assessment in Subjects With Type 2 Diabetes Mellitus. General Population-Based Scores or Specific Scores? Rev Esp Cardiol. 2004;57(6):577-580

5. Herath M Meththananda Herath, Thilak Priyantha Weerarathna, Dilini Umesha. Cardiovascular risk assessment in type 2 diabetes mellitus: comparison of the World Health Organization/International Society of Hypertension risk prediction charts versus UK Prospective Diabetes Study risk engine. Vasc Health Risk Manag. 2015;11:583-589.

6. Marilia B Gomes, Daniel Giannella-Neto, Manuel Faria, et al. Estimating cardiovascular risk in patients with type 2 diabetes: a national multicenter study in Brazil. Diabetol Metab Syndr. 2009;1:22.

7. Joep van der Leeuw, Joline WJ Beulens, Susan van Dieren, et al. Novel Biomarkers to Improve the Prediction of Cardiovascular Event Risk in Type 2 Diabetes Mellitus. J Am Heart Assoc. 2016;5(6).

8. Bulugahapitiya U, Siyambalapitiya S, Sithole J, et al. Is diabetes a coronary risk equivalent? Systematic review and meta-analysis. Diabetic Medicine. 2009;26(2):142-148.

9. Dhakshinamurthy Vijay Anand, Eric Lim, David Hopkins, et al. Risk stratification in uncomplicated type 2 diabetes: prospective evaluation of the combined use of coronary artery calcium imaging and selective myocardial perfusion scintigraphy. Eur Heart J. 2006;27(6):713-721.

10. American Diabetes Association. Complications of diabetes in the United States. 